\title{
The complete mitochondrial genome of Ophiocordyceps gracilis and its comparison with related species
}

\author{
Aifeire Abuduaini ${ }^{1 \dagger}$, Yuan-Bing Wang ${ }^{2 \dagger}{ }^{,}$Hui-Ying Zhou ${ }^{1}$, Rui-Ping Kang ${ }^{1}$, Ming-Liang Ding ${ }^{3}$, Yu Jiang ${ }^{4}$, \\ Fei-Ya Suo ${ }^{1}$ and Luo-Dong Huang ${ }^{4,5^{*}}$ [0
}

\begin{abstract}
In this study, the complete mitochondrial genome of $O$. gracilis was sequenced and assembled before being compared with related species. As the second largest mitogenome reported in the family Ophiocordycipitaceae, the mitogenome of O. gracilis (voucher OG201301) is a circular DNA molecule of 134,288 bp that contains numerous introns and longer intergenomic regions. UCA was detected as anticodon in tRNA-Sec of $O$. gracilis, while comparative mitogenome analysis of nine Ophiocordycipitaceae fungi indicated that the order and contents of PCGs and rRNA genes were considerably conserved and could descend from a common ancestor in Ophiocordycipitaceae. In addition, the expansion of mitochondrial organization, introns, gene length, and order of $O$. gracilis were determined to be similar to those of $O$. sinensis, which indicated common mechanisms underlying adaptive evolution in $O$. gracilis and 0 . sinensis. Based on the mitochondrial gene dataset (15 PCGs and 2 RNA genes), a close genetic relationship between $O$. gracilis and $O$. sinensis was revealed through phylogenetic analysis. This study is the first to investigate the molecular evolution, phylogenetic pattern, and genetic structure characteristics of mitogenome in O. gracilis. Based on the obtained results, the mitogenome of $O$. gracilis can increase understanding of the genetic diversity and evolution of cordycipitoid fungi.
\end{abstract}

Keywords: Mitochondrial genome, Phylogenetic analysis, Ophiocordyceps gracilis, Ophiocordycipitaceae

\section{INTRODUCTION}

Mitochondrion is a semi-autonomous organelle with its own genetic material and genetic system, and it has the ability of independent replication and inheritance. The mitochondrial genome is characterized by a high copy number, rapid evolution, and conservative genetic function (Burger et al. 2003; Bullerwell and Lang 2005; Nadimi et al. 2016). Within ascomycetes, mitochondrial DNA (mtDNA) is often circular and almost all mitochondrial

\footnotetext{
*Correspondence: ynhuangld@gxu.edu.cn

${ }^{\dagger}$ Aifeire Abuduaini and Yuan-Bing Wang have contributed equally to this study

${ }^{4}$ College of Life Science and Technology, Guangxi University, Nanning 530004, China

Full list of author information is available at the end of the article
}

genes are encoded by the same DNA strand (Sandor et al. 2018). Fungal mitogenome normally consists of 14 protein-coding genes (PCGs) including three ATPsynthase subunits, seven NADH dehydrogenase subunits, one complex III (cytochrome c oxidoreductase), and three complex IV (cytochrome c oxidase subunits). Because there are small and large ribosomal RNAs and a set of tRNA genes in the fungal mitogenome (Johnston and Williams 2016; Li et al. 2019b; Wang et al. 2020a), mtDNA has been widely utilized in analyses of molecular evolution, phylogenetic pattern, conservation genetics, population genetics, and genetic structure analyses of fungi (Zhang et al. 2015a; Nadimi et al. 2016; Sandor et al. 2018). original author(s) and the source, provide a link to the Creative Commons licence, and indicate if changes were made. The images or other third party material in this article are included in the article's Creative Commons licence, unless indicated otherwise in a credit line to the material. If material is not included in the article's Creative Commons licence and your intended use is not permitted by statutory regulation or exceeds the permitted use, you will need to obtain permission directly from the copyright holder. To view a copy of this licence, visit http://creativecommons.org/licenses/by/4.0/. 
Owing to the efforts of the Fungal Mitochondrial Genome Project (FMGP), many complete mitogenomes of fungi have been sequenced (Paquin et al. 1997; Husami et al. 2020). Regarding the mitochondrial genomes of Ophiocordycipitaceae (Hypocreales), the nine species thereof have been reported and attracted considerable attention (Wang et al. 2016, 2018; Zhang et al. 2015b, 2017a,b; Huang et al. 2017; Kang et al. 2017; Li et al. 2020a; Zhang and Zhang 2020). Research on the Ophiocordycipitaceae mitogenome provided new insights into the systematics and evolution of entomopathogenic fungi, with the size, structure, order, and content of mitogenomes from these species being highly variable. The mitogenome content is typically conserved, but the mitogenome size and gene synteny still vary $(\mathrm{Li}$ et al. 2020b). Thus far, a detailed comparison of the mitogenome information has not yet been elucidated, which limits the overall understanding of the phylogenetics and adaptive evolution of Ophiocordycipitaceae species.

Among Ophiocordycipitaceae, Ophiocordyceps is morphologically diverse and is the largest genus of the entomopathogenic fungi (Sung et al. 2007). Ophiocordyceps sinensis is well-established for its biological functions against diseases and health benefits in humans, and it is extensively used in traditional Chinese medicine (Marioni et al. 2008; Liang 2018). Of note, the bioactive compounds of $O$. gracilis and $O$. sinensis are almost the same such as D-Mannitol, polysaccharide, adenosine, amino acids, and mannitol. This presents common features for efficient utilization and research into the aforementioned Ophiocordyceps species. Environment heterogeneity studies have revealed that $O$. gracilis grows in coniferous forests at 1116-2076-m altitude and is widely distributed in Russia, Guatemala, Mexico, Norway, Denmark, France, Nepal, India, Mongolia, Kazakhstan, and China (Sung et al. 2007). Ophiocordyceps sinensis parasitizes various ghost moth larvae (Lepidoptera: Hepialidae) and occurs in more diverse habitats (alpine meadow and shrub) in the QinghaiTibetan plateau at 3000-m or higher altitude ( $\mathrm{Li}$ et al. 2011). Hence, habitats can be employed as a useful feature to distinguish $O$. gracilis and $O$. sinensis.

Initially, O. gracilis has been referred to as Xylaria gracilis, which belongs to Xylariaceae. In 1883, the name was revised to Cordyceps gracilis. In the recent phylogenetic classification of insect pathogens of clavicipitaceous fungi, O. gracilis (voucher number EFCC 3101, EFCC 8572 ) was assigned to the $O$. ravenelii clade and was clustered with O. heteropoda. However, O. gracilis (voucher number OSC 151906) is similar to Ophiocordyceps sp. (voucher number OSC151907) within the O. ravenelii clade (Sung et al. 2007; Quandt et al. 2014), which suggests that $O$. gracilis is not monophyletic. The $O$. gracilis representative vouchers were clustered in different clades and could have resulted in a case of mistaken identity. The morphological characteristics and small number of genes of $O$. gracilis can be affected by the environment, and difficulties exists in accurately identifying this species using traditional taxonomic methods. Accordingly, further studies are needed to verify the phylogenetic position of O. gracilis.

The fungus $O$. gracilis produces compounds with numerous biological and pharmacological properties; yet there is a scarcity of genome and genetic information thereof to clarify the evolutionary relationship. In this study, the complete mitogenome of $O$. gracilis (voucher OG201301) was assembled and annotated. The content, structure, and gene order of the mitogenome of $O$. gracilis were analyzed to deepen the understanding of its mitogenome structure. Based on the characteristics of nine fungal mitogenomes of Ophiocordycipitaceae, the differences between them were compared. Meanwhile, a detailed comparative mitogenomic analysis was conducted on $O$. sinensis, which is a typical entomopathogenic fungus and the largest mitogenome in Ophiocordycipitaceae. Ultimately, phylogenetic analysis adopting complete mitochondrial data provides a theoretical basis for the conservation and sustainable utilization of $O$. gracilis resources and furthers the current understanding of Hypocreales fungi in terms of systematics and evolutionary biology.

\section{MATERIALS AND METHODS}

\section{Sampling, DNA extraction, and NGS sequencing}

O. gracilis voucher OG201301 was collected from the Altai Mountains of Xinjiang, China. The asexual strain of OG201301 was isolated as described in a previous study (Wang et al. 2020b). The voucher OG201301 was stored in the fungarium of our laboratory at Guangxi University, and the culture (KUMCC 3001) isolated from that voucher was deposited in the public Culture Collection of the Kunming Institute of Botany (KUMCC). Axenic living cultures were used for genomic DNA extraction using a Plant Genomic DNA Extraction Kit (Takara, Biotech Co., Beijing, China). A total of $5 \mu \mathrm{g}$ micrograms of genomic DNA from O. gracilis was sheared into $270 \mathrm{bp}$ fragments as the DNA library. The library was carried on the Illumina HiSeq 4000 platform for paired-end 150 bp sequencing by BGI Biotechnology Co. (Shenzhen, China). The generated DNA raw data was stored in the NCBI Short Read Archive (SRA) under accession number PRJNA627096.

\section{De novo assembly and annotation of the mitogenome}

To eliminate adaptor sequences, reads with low-quality sequences, and unknown nucleotides, the raw reads 
were filtered after sequencing. SPAdes 3.9.0 was used to assemble high-quality clean reads (Bankevich et al. 2012). The assembled contig sequences were aligned using BLAST against the $\mathrm{Nr}$ database to screen out mitochondrial contigs. One contig of mitochondrial DNA was identified on the basis of sequence similarity to other Ophiocordycipitaceae mitogenomes and the contig gaps were filled through sequence extension and iterative mapping method by PRICE (Paired-Read Iterative Contig Extension) (Ruby et al. 2013). Finally, mitogenome annotation was performed using methods proposed by Li et al. (2019b). The mitogenome was automatically annotated using the MFannot tool (https://megasun.bch.umontreal. ca/cgi-bin/RNAweasel/RNAweaselInterface.pl) based on the mold mitogenome genetic code (Valach et al. 2014). Subsequently, the UGENE ORFs finder was adopted to identify protein-encoding genes (Okonechnikov et al. 2012). The analysis of tRNA and rRNA genes was conducted using tRNAscan-SE 2.0 (Lowe and Chan 2016) and RNAmmer 1.2 Server (Lagesen et al. 2007), respectively. For the gene-containing introns, exonerate software was applied to compare the amino acid sequences of the near-source species to determine the boundaries and lengths of the introns. The introns were detected by RNAweasel (http://megasun.bch.umontreal.ca/cgi-bin/ RNAweasel/RNAweaselInterface.pl).

\section{Sequence analysis}

The complete mitogenome sequence along with gene annotations of $O$. gracilis was deposited in the GenBank database under the accession numbers of MT371080, while the physical genome map was drawn using the online software GenomeVx (Conant and Wolfe 2008). The Unipro UGENE was employed to analyze all mitogenomes for the based composition thereof. The following formulas were employed to access mitogenome strand asymmetry: A-T skew $=[\mathrm{A}-\mathrm{T}] /[\mathrm{A}+\mathrm{T}]$ and $\mathrm{G}-\mathrm{C}$ skew $=[\mathrm{G}-\mathrm{C}] /[\mathrm{G}+\mathrm{C}]$. The codon usage was analyzed and visualized using MEGA 7.0 (Kumar et al. 2016). The Mauve 2.3.1 was applied to determine the collinearity of mitogenomes between $O$. gracilis and eight other fungi from Ophiocordicipitaceae (Darling et al. 2004). Subsequently, Circoletto was applied to identify the interspersed repeats through the O. gracilis and O. sinensis mitogenomes, the E-value of which was $10^{-5}$ (Darzentas 2010). The repeated sequences were detected by REPuter, in which the default parameters were used (Kurtz et al. 2001). The tandem repeats were located using the Tandem Repeats Finder (https://tandem.bu.edu/trf/trf.basic. submit.html), and simple sequence repeats (SSRs) were distinguished using the online MIcroSAtellites classification tool (Beier et al. 2017).

\section{Phylogenetic analysis}

To investigate the phylogenetic position of $O$. gracilis in the family Ophiocordicipitaceae, a phylogenetic tree of 24 Hypocreales fungi premised on the allocated sequential gene set (14 core PCGs + rps3 + 2 RNA genes) was constructed. For the analysis of homologous gene set sequence, TBtools was used to perform gene sequence extraction and local blast alignment (Chen et al. 2020). The mitochondrial gene set was aligned using RAxML v8.0.0 and MAFFT v7.037 to construct a phylogenetic tree (Stamatakis 2014; Katoh et al. 2017) for which the Bayesian inference (BI) method was adopted. MrBayes v.3.1.2 was applied to conduct BI analysis for three million generations using the most appropriate model, with 1000 bootstrap replicates (Ronquist et al. 2012).

\section{RESULTS AND DISCUSSION}

\section{Mitogenomic characterization of Ophiocordyceps gracilis}

The complete mitogenome of $O$. gracilis was assembled as a circular molecule 134288 bp long (Fig. 1; Additional file 2: Table S1), and it was the second largest mitogenome reported among the Ophiocoedycipitaceae (Table 1). In general, mitogenomic coding is associated with the mitochondrial translation apparatus, electron transport, and oxidative phosphorylation (Burger et al. 2003).

The PCGs of $O$. gracilis included 15 PCGs related to oxidative phosphorylation [NAD dehydrogenase (nad1-6 and nad4L), cytochrome oxidases (cox1-3), cytochrome b (cob), and three ATP synthases (atp6, atp8 and atp9), ribosomal protein S3 (rps3)], 4 hypothetical proteins (orf751, orf272, orf172 and orf221), 2 ribosomal RNA genes (rns and $\mathrm{rnl}$ ), and 24 transfer RNA genes (Additional file 2: Table S1). In the mitogenome of O. gracilis, the length of 15 PCGs varied from 147 bp (atp8) to $19,137 \mathrm{bp}$ (cox1). The total length was $15,996 \mathrm{bp}$, which accounted for $11.91 \%$ of the mitogenome. Being $1.35 \%$ of the mitogenome, the length of tRNA was $1813 \mathrm{bp}$, while the length of rRNAs was $5253 \mathrm{bp}$, which was 3.91\% of the mitogenome. Further, the length of intergenic regions (IRs) was $30,299 \mathrm{bp}$, which accounted for $22.56 \%$ (Table 1). Thus, the generated length of $O$. gracilis varied through 15 PCGs, which contributed to mitogenome expansion. The assumption was that the protein-coding regions could be additional factors for the large size of the fungal mitochondria.

The GC concentration of the mitogenome differs among species, which may be affected by mutation bias, selection, and biases of reconstitution-related DNA repair (Li et al. 2018). The nucleotide composition of $O$. gracilis was biased towards AT (71.35\%) and the GC content was $28.65 \%$. According to the second parity rule of Chargaff (A-T and G-C within one strand), O. gracilis 


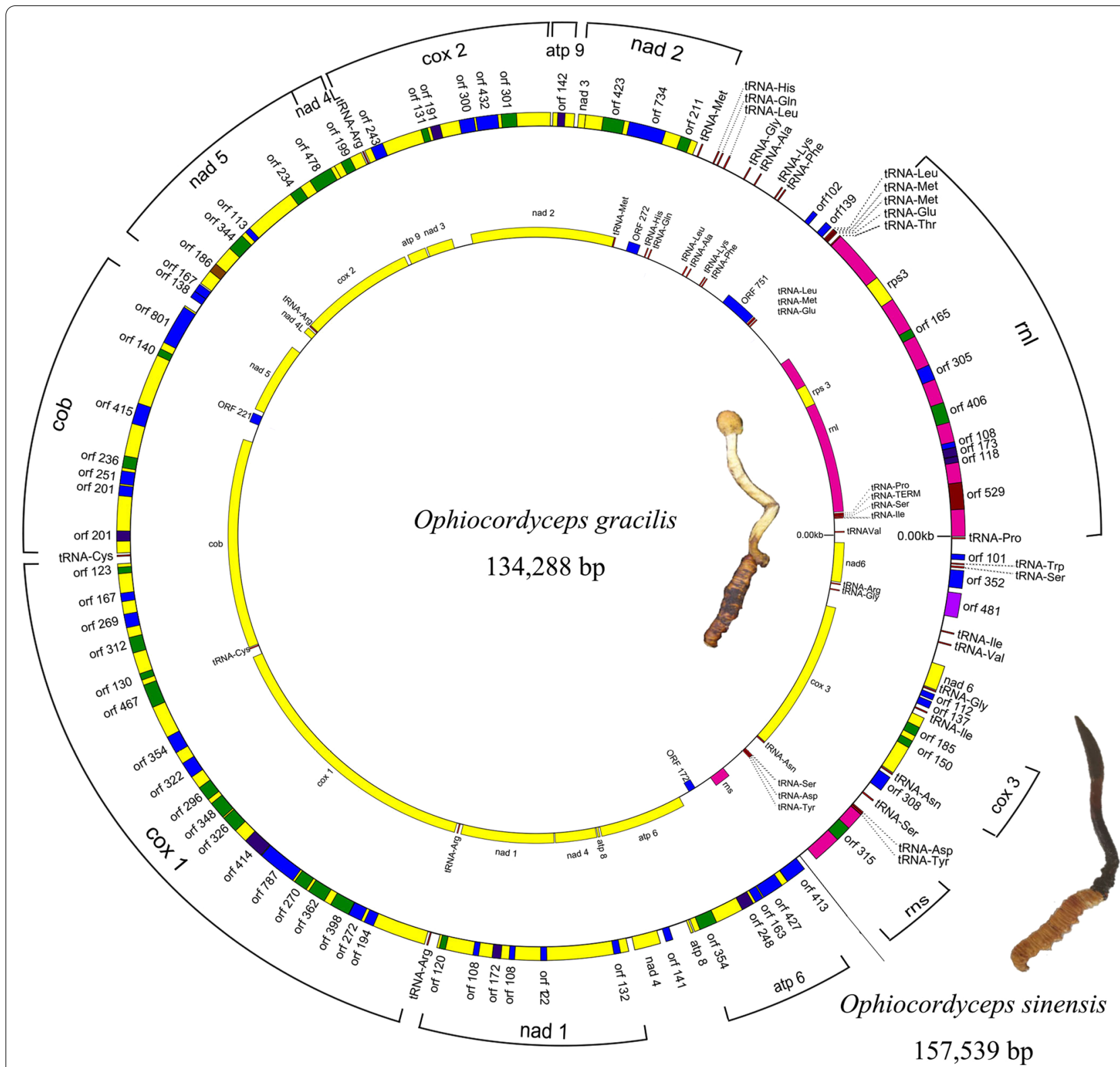

Fig. 1 Mitogenome map of Ophiocordyceps gracilis and O. sinensis. The outer layer represents O. sinensis obtained from Kang et al. (2017); the inner layer represents $O$. gracilis from this study. The genes are shown in different color blocks

was maintained at the same frequency (Chen et al. 2014), where an observation could be made that GC and AT skews therein were positive with AT-skew $<1$ (Additional file 2: Table S1).

Aside from nad4 that was initiated with ATC (Table 2), PCGs were initiated with typical ATG start codons. The remaining PCGs of atp6, cox3, and nad6 start codons were TTA. The majority of PCGs were terminated by typical TAA codons, except for rps3, nad2 and cox1, with two genes terminal codons being TAG. All protein and RNA genes were encoded in positive strands except for orf172 (Additional file 2: Table S1). Similarly, the rps3 coding ribosomal protein S3 was included in rnl (Fig. 1 and Additional file 2: Table S1). Only tRNA-Met was connected to tRNA-Leu, and the longest distance was $3462 \mathrm{bp}$ (tRNA-Glu and rnl), with 8 tRNAs being on both sides of rnl (Additional file 2: Table S1). This result indicates that the mitogenome of $O$. gracilis has non-continuous segments, abundant introns, and lack of overlapping genes (Additional file 2: Table S1).

Selenocysteine $(\mathrm{Sec})$ was the 21st discovered amino acid, and UGA is the dominant Sec codon in use 


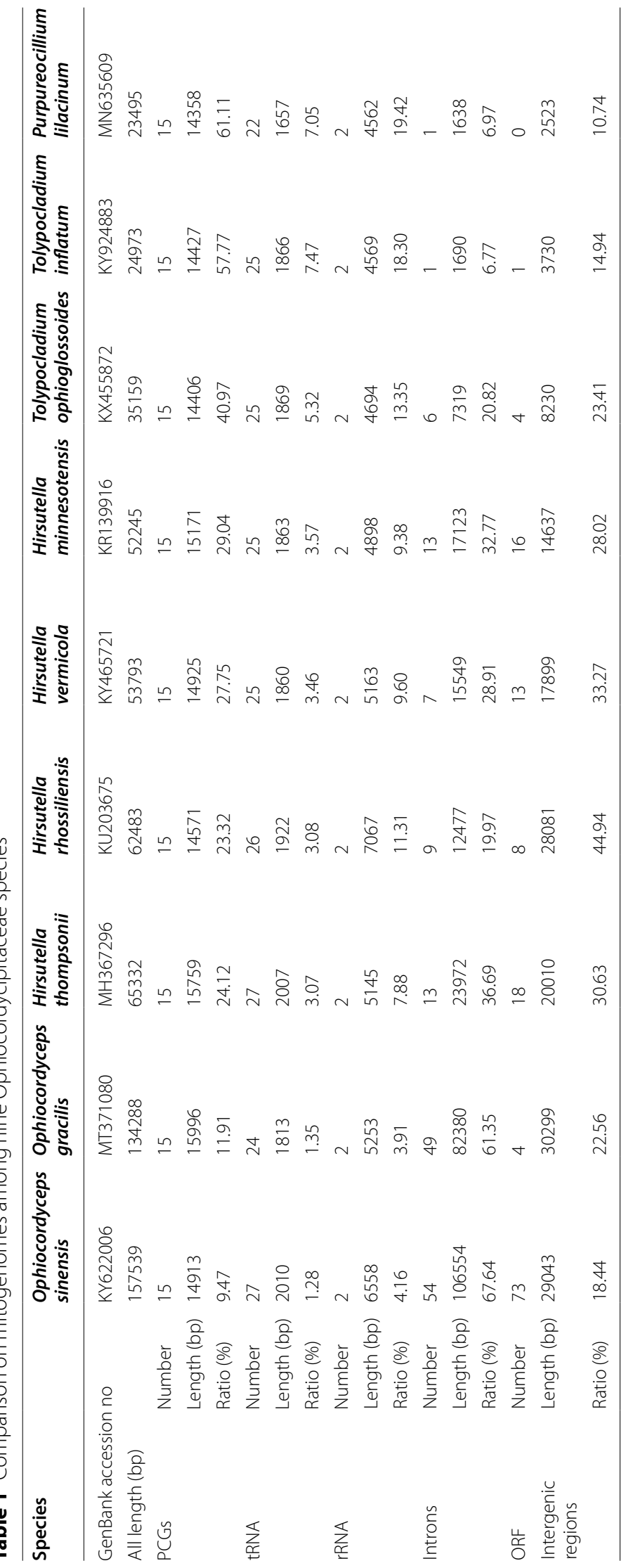


Table 2 PCGs and rRNA genes composition of Ophiocordyceps gracilis mitogenome

\begin{tabular}{|c|c|c|c|c|c|c|c|}
\hline Genes & Location & Start/stop codon & $\begin{array}{l}\text { Introns } \\
\text { number }\end{array}$ & Introns length & Exon length & Strand & $\begin{array}{l}\text { Coding } \\
\text { sequence } \\
\text { percentage (\%) }\end{array}$ \\
\hline $\mathrm{rnl}$ & $1655-13038$ & - & 5 & 7261 & 4123 & + & 36.22 \\
\hline rps3 & $9424-10875$ & ATG/TAG & 0 & 0 & 1452 & + & 100 \\
\hline nad2 & 28088-38134 & ATG/TAG & 5 & 8112 & 1935 & + & 19.26 \\
\hline nad3 & $39492-41342$ & ATG/TAA & 1 & 1437 & 414 & + & 22.37 \\
\hline atp9 & $41459-42766$ & ATG/TAA & 1 & 1083 & 225 & + & 17.20 \\
\hline $\cos 2$ & $43009-51117$ & ATG/TAA & 4 & 7356 & 753 & + & 9.29 \\
\hline nad4L & $51358-51780$ & ATG/TAA & 0 & 0 & 423 & + & 100 \\
\hline nad5 & 53097-58109 & ATG/TAA & 3 & 2958 & 2055 & + & 40.99 \\
\hline$c o b$ & $60382-75098$ & ATG/TAA & 8 & 13544 & 1173 & + & 7.97 \\
\hline $\operatorname{cox} 1$ & $75840-94976$ & ATG/TAG & 10 & 16566 & 2571 & + & 13.43 \\
\hline nad1 & 95433-101968 & ATG/TAA & 3 & 5426 & 1110 & + & 16.98 \\
\hline nad4 & 102050-104962 & ATC/TAA & 1 & 1449 & 1464 & + & 50.26 \\
\hline atp8 & $105038-105184$ & ATG/TAA & 0 & 0 & 147 & + & 100 \\
\hline atp6 & 105309-111399 & TTA/TAA & 2 & 5317 & 774 & + & 12.71 \\
\hline rns & $114044-115173$ & - & 0 & 0 & 1130 & + & 100 \\
\hline $\operatorname{cox} 3$ & 118592-129212 & TTA/TAA & 5 & 9820 & 801 & + & 7.54 \\
\hline nad6 & 131019-133768 & TTA/TAA & 1 & 2051 & 699 & + & 25.42 \\
\hline
\end{tabular}

(Vargas-Rodriguez et al. 2018); the Sec-UGA codon is a controversial stop codon (Serrão et al. 2018); In O. gracilis, UCA is an anticodon in tRNA-Sec, which indicates that UCA-anticodon is involved in the specificity and adaptive evolution thereof.

The tRNA had the same significance during translation as mRNA and proteins (Kirchner and Ignatova 2014). In O. gracilis, 24 tRNAs genes in the mitogenome encoded the 20 standard amino acids, while tRNA-Trp and tRNAThr were not predicted, with a range of 71-87 bp (Additional file 3: Table S2). The difference in the predicted tRNA secondary structure can be ascribed to the different analysis methods adopted. In addition, 19 tRNA exhibited typical clover-leaf secondary structure, i.e., tRNA-Ser, tRNA-Leu, and tRNA-Tyr with a variable loop. All tRNAs exhibited typical clover-leaf secondary structure, and the remaining mismatched bases had non-canonical G-U pairs (Fig. 2). In accordance with the analysis of the secondary structure in fungi, the variations in the numbers of extra arms may cause differences in tRNA length. Further, in O. gracilis, there was a lack of tRNA-Thr and tRNA-Trp, while there was one less tRNA-Met and one more tRNA-Arg compared to other fungi (Additional file 4: Table S3).

\section{Comparative mitogenomics of Ophiocordycipitaceae fungi} The nine published fungal mitogenomes of Ophiocordycipitaceae were compared, for which consideration was given to the fact that variation in fungal mitogenomes occurs in gene order, genome size, introns, ORFs, and intergenic regions. Mitogenome sizes of nine fungi were significantly different, with the sizes ranging from 23,495 bp to 157,539 bp (Fig. 3; Table 1). Mitogenomic lengths of Hirsutella rhossiliensis, $H$. vermicola, $H$. minnesotensis, $H$. thompsonii, Tolypocladium inflatum, $T$. ophioglossoides, and Purpureocillium lilacinum were an order of magnitude smaller than O. gracilis and O. sinensis. The mitogenome of $O$. sinensis was the maximum length reported for Ophiocordycipitaceae, which was similar to that of O. gracilis (Fig. 3; Table 1). In particular, the introns and non-coding regions were similar in $O$. gracilis and $O$. sinensis (Fig. 1).

The number and size of introns in nine Ophiocordycipitaceae fungi were measured and compared. (1) The largest was $O$. sinensis, which contained 54 introns with a total size of 106,554 bp. (2) O. gracilis was the second largest, and contained 49 introns totaling $82,380 \mathrm{bp}$. (3) H. minnesotensis contained 13 introns totaling 17,123 bp. (4) H. thompsonii contained 13 introns totaling 23,972 bp. (5) H. rhossiliensis had 9 introns totaling $12,477 \mathrm{bp}$. (6) $\mathrm{H}$. vermicola had 7 introns totaling 15,549 bp. (7) T. ophioglossoides had 6 introns totaling 7913 bp. (8) T. inflatum had 1 intron of $1690 \mathrm{bp}$. (9) P. lilacinum was the smallest and contained, 1 intron totaling $1638 \mathrm{bp}$ (Table 1).

The synteny results in Mauve revealed that the homologous collinear blocks occurred in all nine fungal mitogenomes of Ophiocordycipitaceae (Fig. 3). An observation could be made that nine species were 


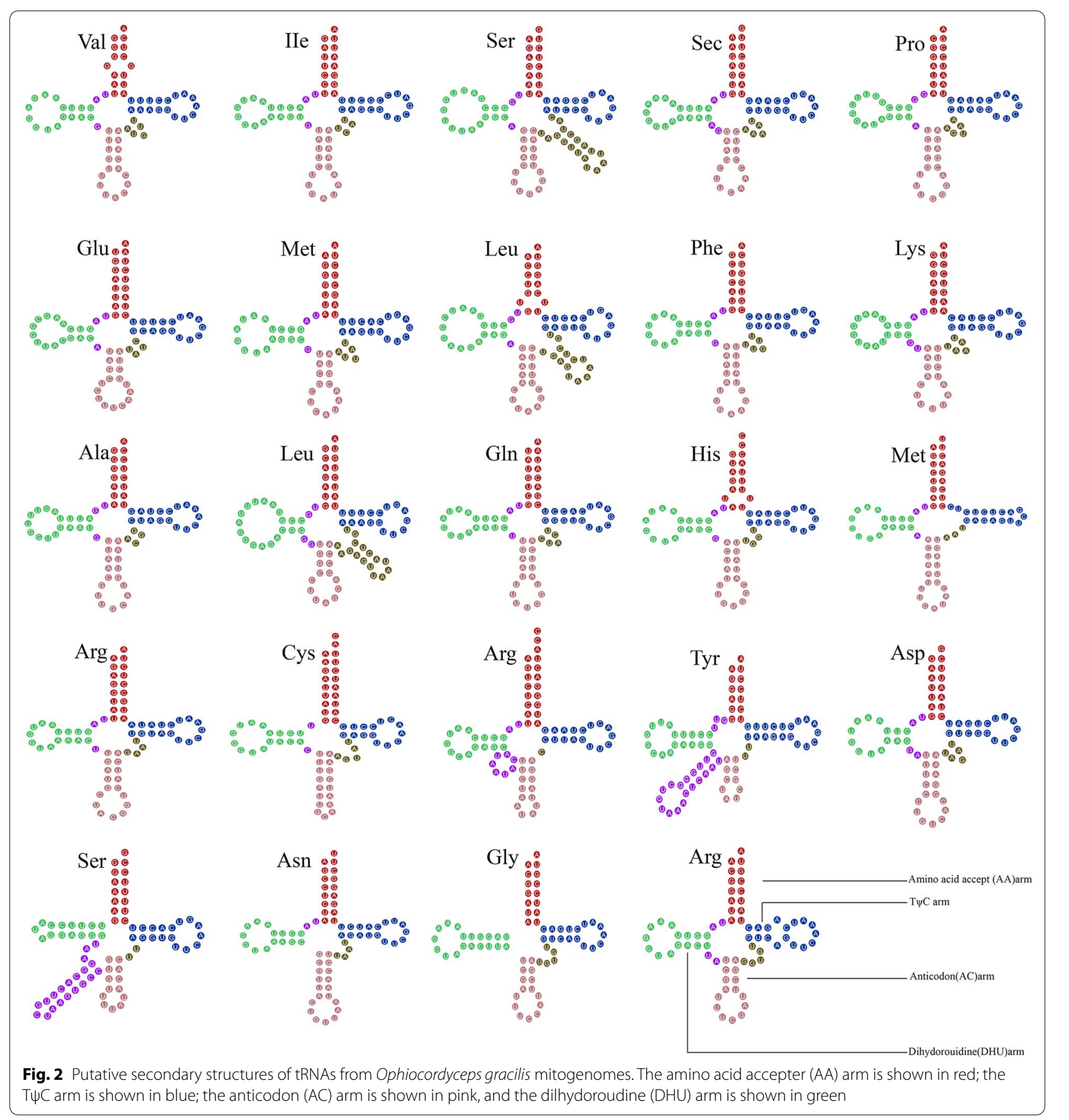

highly similar in PCGs, ribosomal RNA, and tRNA gene composition and quantity. The 15 PCGs and 2 rRNA genes of the nine species were arranged as rnl (rps3) -nad2 -nad3 -atp9 -cox2 -nad4L -nad5 -cob -cox1 -nad1 -nad4 -atp8 -atp6 -rns -cox3 -nad6. All genes were arranged in the same order as the gene arrangement of Ophiocordycipitaceae (Additional file 4: Table S3). At the synteny level, the mitogenome of $O$. gracilis was conserved in the gene arrangement and gene order of 15 PCGs, 2 rRNA genes and the majority of tRNA genes of Ophiocordycipitaceae. Owing to the high evolution rate, mitogenomic introns can differ between species within the same genus or even intraspecies (Deng et al. 2016). Of note, the mitogenome of $O$. sinensis contains the largest number of introns and intergenic regions, and O. gracilis is the second largest. 


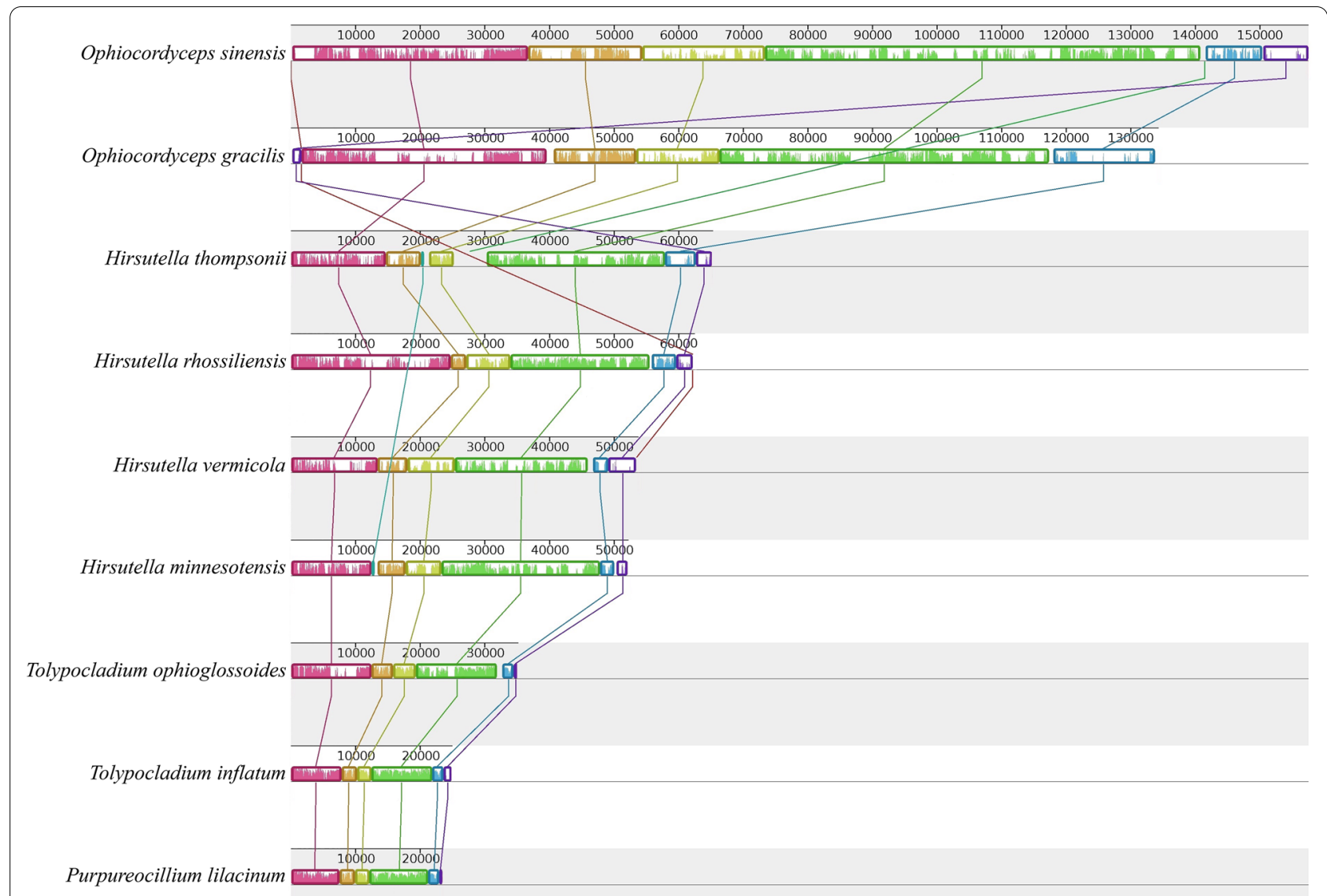

Fig. 3 Collinearity analysis of nine mitogenomes from Ophiocordycipitaceae. Homologous regions between different mitogenomes are represented by the same color blocks linked by lines

Therefore, the increased size of mitogenomes may be the result of intergenic region expansion.

Ophiocordyceps gracilis was similar to O. sinensis, and both had gene rearrangements and homologous regions in the mitogenome (Additional files 1: Fig. S1, 2: Table S1). The results of Circoletto analysis indicate that the similarity of mitogenomes of $O$. gracilis and O. sinensis was $87 \%$ (Fig. S1) with bases containing 51,041 bp. Multiple introns and intergenic regions were present in the mitogenomes of $O$. gracilis and $O$. sinensis, which differed in location, number, and length.

An in-depth comparison was conducted on the differences and similarities between $O$. gracilis and $O$. sinensis. Unlike O. sinensis, nad2/nad3 genes and nad4L/nad5 in O. gracilis were connected, and an overlap of base "A" was also observed (Li et al. 2015; Kang et al. 2017) (Fig. 1; Additional file 2: Table S1). The differences included varying lengths of 15 PCGs across the mitogenomes of $O$. gracilis and O. sinensis, in which cox $1, \operatorname{cox} 2, \operatorname{cox} 3$, nad1, nad2, nad3, nad4, nad4L, nad5, and nad6 exhibited differences in length, while atp8 and atp9 were the same (Fig. 4a). The GC content in the O. gracilis mitogenome was $28.65 \%$, which was lower than that in the O. sinensis mitogenome (30.2\%). The GC content of 15 PCGs varied across the mitogenomes. The nad2 contained the highest GC content in both O. gracilis and O. sinensis $(31.09 \%$ and $31.58 \%$, respectively) (Fig. 4b). There was a positive AT skew of atp6, atp9, cob, cox1, cox2, cox3, nad1, nad2, nad3, nad6, and rps3, while atp8, nad4, nad4L, and nad5 had a negative A-T skew. Most PCGs exhibited a positive A-T skew in O. gracilis and O. sinensis. In addition, nad4L and nad5 exhibited a negative A-T skew in O. gracilis and a positive A-T skew in O. sinensis.

A negative A-T skew was observed in atp8 and nad4 while a positive A-T skew is present in nad3 and nad6 in O. graciliis (Fig. 4c). Most PCGs exhibited a positive G-C skew and only atp8 exhibited a negative G-C skew. The $\mathrm{GC}$ content of $O$. gracilis was higher than that of $O$. sinensis at atp6, atp8, atp9, cob, cox1, cox2, cox3, nad6, and rps3 (Fig. 4d).

The relative synonymous codon usage (RSCU) values of O. gracilis and O. sinensis are outlined in Fig. 5 and Additional file 5: Table S4. The pattern of codon usage was generally similar among the fungal mitochondria, 

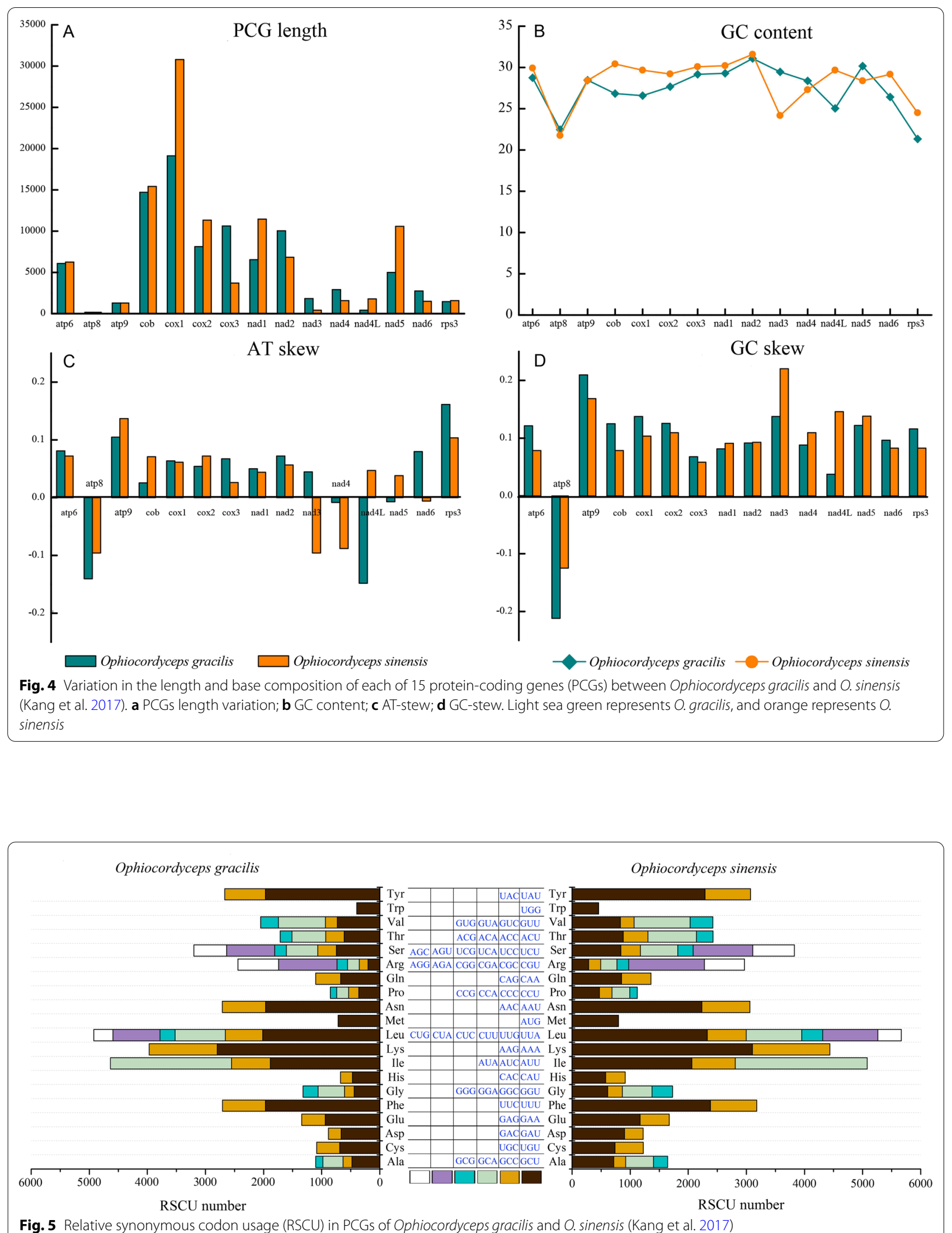

Fig. 5 Relative synonymous codon usage (RSCU) in PCGs of Ophiocordyceps gracilis and O. sinensis (Kang et al. 2017) 
including the most frequently used codons UUU, UUA, CUU, AUU, AUA, AUG, GUU, GUA, and 30 other codons, except for UAA. Based on the codon usage analysis, the most used codons in the two mitogenomes were UUU (for Phe), AUU and AUA (for IIe), AAA (for Lys), UUA (for Leu), AAU (for Asn), and UAU (for Tyr). The high frequency of $\mathrm{A}$ and $\mathrm{T}$ use in codons was observed (Additional file 5: Table S4).

Four ORFs were predicted in O. gracilis, while 73 ORFs were predicted in $O$. sinensis (Additional file 2: Table S1). The lengths of ORFs were 2256 bp (orf751), 819 bp (orf272), 666 bp (orf221), and 519 bp (orf172). The mitogenome of $O$. gracilis demonstrated variation in the relative positions and sizes of ORFs. Conversely, the mitogenome of $O$. sinensis included hypothetical protein (35), LAGLIDADG endonuclease (27), GIY-YIG endonuclease (8), RNA-dependent DNA polymerase (1), RNA polymerase (1), and GIY-YIG/LAGLIDA-DG (1).

The comparative analysis of tRNA genes in the O. gracilis and O. sinensis mitogenomes show that $O$. gracilis contains two copies of tRNA-Ser and tRNA-Leu, and three copies of tRNA-Arg, while there were two copies of tRNA-Leu, tRNA-Gly, tRNA-Arg, tRNA-IIe, and tRNASer and three copies of tRNA-Met present in O. sinensis. These results suggest that one copy of tRNA-Gly, and tRNA-IIe may have been lost in O. gracilis or tRNA-Arg was duplicated in O. sinensis (Additional file 2: Table S1). Hence, an observation could be made that sequence differences in tRNA genes were associated with variation, which was central to phylogenic and genetic relationship.

\section{Identification of repetitive elements in 0 . gracilis and $O$. sinensis}

Bibiserv-REPuter was applied to detect repeated sequences, it can locate and identify forward, reverse, complementary and palindromic repeats (Kurtz et al. 2001). To compare the differences between O. gracilis and $O$. sinensis, the default parameter settings were used, with the maximum computed repeats and minimal repeat size being limited to 50 and 8 , respectively. In the mitogenome of $O$. gracilis, REPuter identified 32 forward types (a total of $3998 \mathrm{bp}$ ), 11 palindromic types (a total of $1400 \mathrm{bp}$ ), and 7 of reverse repeat types (a total of $780 \mathrm{bp}$ ). Meanwhile, 32 forward types (a total of $5158 \mathrm{bp}$ ), 14 palindromic types (a total of $2064 \mathrm{bp}$ ), and 4 reverse repeat types (a total of $556 \mathrm{bp}$ ) were identified in $O$. sinensis (Additional file 6: Table S5). The Tandem Repeats Finder was used to detect 48 and 44 tandem repeats in O. gracilis and O. sinensis, respectively (Additional file 7: Table S6). The longest tandem sequence was observed in the mitogenomes of O. gracilis (57 bp) and O. sinensis (123 bp). Most tandem repeat sequences were copied in the two species. Tandem repeat sequences accounted for
$1.93 \%$ and $1.76 \%$ of the mitogenome lengths of $O$. gracilis and $O$. sinensis, respectively.

Interspersed repeats and partial tandem repeats (microsatellites and minisatellites) are moderately repetitive sequences. As simple sequence repeats (SSRs) or short tandem repeats (STR), the basic component of a microsatellite is a short sequence motif (1-6 bp in length) repeated in tandem ( $\mathrm{Li}$ et al. 2018). Microsatellites are ubiquitous and highly variable across all eukaryotic groups. In general, the microsatellite frequency is positively correlated with the genome size (Garner 2002). A microsatellite identification tool detected SSRs numbers of 46 and 43 in O. gracilis and O. sinensis, respectively (Additional file 8: Table S7). The majority of these SSRs sequences were rich in $\mathrm{A} / \mathrm{T}$, and 31 and 36 of $\mathrm{A} / \mathrm{T}$ were in the mitogenome of $O$. gracilis and $O$. sinensis, respectively. Thus, the rapid expansion in mitogenomes of $O$. gracilis and $O$. sinensis was promoted by repeats and duplicates.

\section{Expansion of intron in O. gracilis and O. sinensis}

Common in fungal mitochondria and occurring in mitochondrial PCGs and rRNA genes, introns are ideal tools to investigate genetic variation within fungi and to identify different species (Sosa-Gómez et al. 2009; Xu et al. 2009; Qin et al. 2010; Ferandon et al. 2013; Zhang and Zhang 2019). The accumulation, movement, and degeneration of introns can effectuate intronic polymorphism in closely related species on an intraspecific level. The gain and loss of introns in these conserved genes, including $\mathrm{rnl}$ and rns, may occur by horizontal gene transfer (Lang et al. 2007). Premised on the secondary RNA structures and splicing mechanism, these introns are primarily categorized into Groups I or II (Saldanha et al. 1993; Lang et al. 2007); Group I introns are relatively more abundant in fungal mitogenomes (Zhang and Zhang 2019).

A total of 49 introns were detected in the O. gracilis mitogenome, with 12 in PCGs and 1 site in rRNA (rnl) (Additional file 9: Table S8). In the O. gracilis mitogenome, 37 introns were categorized into Group I, 3 were in Group II, and 9 introns were not classified. Within Group I, the introns were further subdivided into IA (with 7 introns), IB (19), IC (8), and ID (3). IB was the largest subgroup, which was also the same in the other fungi (Li et al. 2015; Zhang et al. 2015b; Kang et al. 2017; Wang et al. 2018).

The $O$. gracilis introns were identified in $\mathrm{rnl}$, nad2, nad3, atp9, cox2, nad5, cob, cox1, nad1, nad4, atp6, cox3, and nad6. Introns were most common in cox1 (10 sites) and cob (8 sites); rnl, nad2, and cox 3 all had 5 sites; cox 2 had 4 sites; nad5 and nad1 had 3 sites; atp6 had 2 sites; nad3, atp9, nad4, and nad6 were only identified in 1 site; and no introns were identified in nad4L, atp8, and rns. 
In O. sinensis, there was no indication of nad3, nad4, and atp8. However, rns was detected across 8 sites in $O$. sinensis. The introns in $\mathrm{rnl}$ accounted for $63.78 \%$ and PCGs accounted for $84.32 \%$. The introns of $O$. gracilis had an overall length of $82,380 \mathrm{bp}$ and constituted $61.35 \%$ of the entire mitogenome (Table 1). Combined with the analysis of $O$. gracilis and several other fungi, an assumption could be made that, for most fungal species, Group I introns may be included in the rps3 gene within the large subunit ribosomal RNA (rnl) gene (Zhang et al. 2017a,b; Wang et al. 2018).

The number, size, gene content, and insertion site vary among species with a close association with each other and also within the same species (Jung et al. 2012; Torriani et al. 2014; Jalalzadeh et al. 2015; Zhang et al. 2015a; Li et al. 2020b). The genomic information analysis indicates that $O$. gracilis is considerably similar to $O$. sinensis in the family Ophiocordycipitaceae (Table 1), where the number and size of introns in $O$. gracilis are revealed to be the second largest, below those in O. sinensis (Kang et al. 2017). Therefore, an assumption could be made that $O$. gracilis and $O$. sinensis are significantly different from other fungi of Ophiocordycipitaceae, with large mitogenomes and many introns. Mitogenome comparison demonstrates that intron length significantly contributes to the size of the mitogenome (Himmelstrand et al. 2014; Zhang et al 2015a; Deng et al. 2016). Hence, the intron presence/absence dynamics of the O. gracilis and $O$. sinensis mitogenomes can also further the understanding of the evolutionary dynamics of mitochondrial introns.

\section{Phylogenetic analysis}

PCGs and rRNA genes from the mitogenome can also provide useful information to help understand the evolution and phylogeny of fungi (Wang et al. 2018, 2020a). The BI analysis was employed to reveal the phylogenetic position of $O$. gracilis and 23 other species from Hypocreales (Additional file 10: Table S9). On the basis of the combined gene dataset (15 PCGs and 2 rRNA) using the GTR + G + I model (Ronquist et al. 2012), a phylogenetic tree was constructed. All major clades within the tree had sufficient bootstrap support [Bootstrap support $(\mathrm{BS}) \geq 90$ ] (Fig. 6). The 24 species of Hypocreales highly varied in size and were divided into three major clades including the families of

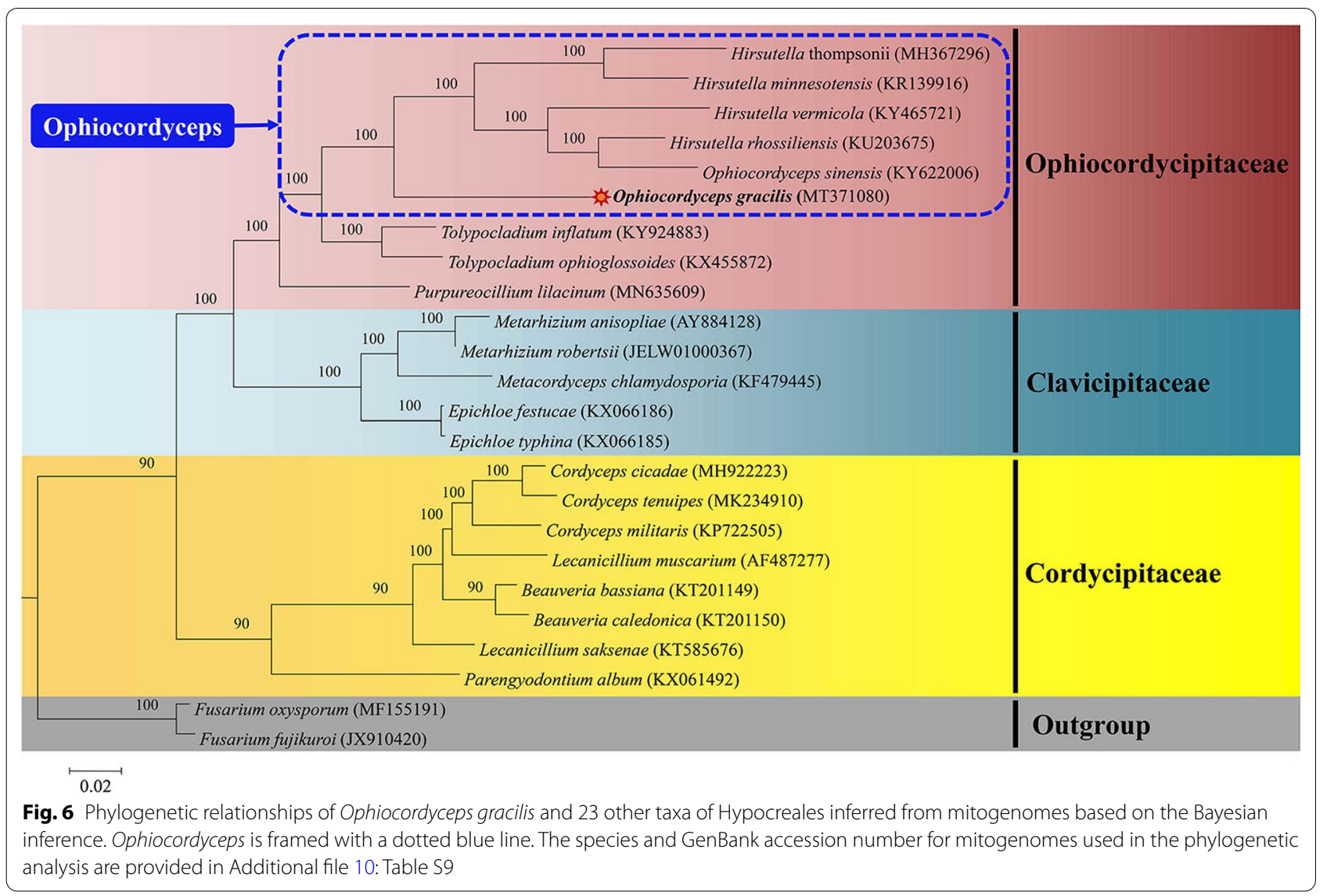


Ophiocordycipitaceae, Clavicipitaceae, and Cordycipitaceae (Additional file 10: Table S9). The results indicated that $O$. gracilis was clustered in the genus Ophiocordyceps of Ophiocordycipitaceae to generate a separate clade with strong statistical support by posterior probabilities (BI-PP $=100 \%)$. Because closeness to the $O$. sinensis clade was exhibited, it was suggested that $O$. gracilis and $O$. sinensis originated from a common ancestor. It was surprising to see rps3 included in $\mathrm{rnl}$, both in $O$. gracilis and $O$. sinensis. Of note, for both O. gracilis and O. sinensis, rps3 is present in rnl, which is different from 14 core PCGs in Hypocreales fungi (Lin et al. 2015; Kang et al. 2017).

To understand the evolution of fungi, mitochondrial genes were adopted in molecular phylogenetics and population genetics research (Nadimi et al. 2016; Dai et al. 2018). Many phylogenetic studies on fungal mitochondria, such as the large subunit gene (rnl), investigate how different organisms are related to each other (Borstler et al. 2008). These analyses seek to identify genes, which provide a clear phylogenetic signal, that differ among closely related taxa and are important in the assessment of the evolutionary relationships of mitogenomes (Nadimi et al. 2016). In this study, the phylogenetic topological structure is consistent with those in prior studies, where inference was made from both BI analysis based on 27 taxa of the Hypocreales order ( $\mathrm{Li}$ et al. 2019a). Mitochondrial genes are useful molecular markers for determining the phylogenetic relationships in the family Ophiocordycipitaceae.

\section{CONCLUSION}

To our knowledge, this study is the first in which the overall characteristics of the mitogenome of $O$. gracilis was sequenced, assembled, annotated, and analyzed. As the second largest reported mitogenome in Ophiocoedycipitaceae, the complete mitogenome of $O$. gracilis was 134,288 bp long, with a non-continuous structure, abundant introns, large intergenic regions, and lack of overlapping genes. The number, length of repeats, duplicates, introns, and PCGs contributed to the size variations in the mitogenome of $O$. gracilis. The genome size, gene content, tRNAs, rRNAs, and collinearity varied among the nine Ophiocordycipitaceae species studied. This difference may indicate that there is variation in introns and intergenic regions. The mitogenome sizes of $O$. gracilis and $O$. sinensis were an order of magnitude higher than those of seven Ophiocordycipitaceae species. In particular, the types and amounts of tRNA, intron, and ORF were significantly different. An observation was made that the mitogenome of $O$. sinensis exhibits similarity to O. gracilis in Ophiocordycipitaceae, especially in terms of introns and non-coding regions. The presence/absence of introns suggests that introns are related to the evolutionary dynamics in the mitogenome.

The mitogenomes were compared to verify the phylogenetic relationship between nine related Ophiocordycipitaceous fungi. Using collinear calculation, all nine species were determined to contain 6 homologous collinear blocks, which maintained the conservation of gene orders at the mitogenome level. The results of comparative mitogenomic analysis indicate that the gene order tended to be conserved, with 14 core PCGs genes and rps3 being invariably retained in nine Ophiocordycipitaceae species. The number of genes was different; in particular, several tRNA had two or three copies, while tRNA-Trp and tRNA-Thr were not predicted in O. gracilis. Further, O. gracilis has tRNA-Sec (UCA), with selenocysteine ( $\mathrm{Sec}$ ) as the $21^{\text {st }}$ amino acid, which may be related to adaptive evolution.

Phylogenetic analysis demonstrates that $O$. gracilis is clustered in the genus Ophiocordyceps of Ophiocordycipitaceae. Ophiocordyceps gracilis is closely related to $O$. sinensis and exhibits a close evolutionary relationship. This study provides new insights on the genetics, systematics, and evolution of $O$. gracilis. In addition, the comparative analysis of nine fungal mitogenomes will enrich understanding of the mitochondrial evolution of Ophiocordycipitaceae.

\section{Abbreviations \\ Mitogenome: Mitochondrial genome; mtDNA: Mitochondrial DNA; BI: Bayes- ian inference; ML: Maximum likelihood; FMGP: Fungal mitochondrial genome project; Nr: Non-redundant protein sequence database; PCGs: Protein-coding genes; tRNA: Transfer RNA gene; rRNA: Ribosomal RNA gene; rnl: Large subunit ribosomal RNA; rns: Small subunit ribosomal RNA; orf: Open reading frame; IRs: Intergenic regions; SSRs: Simple sequence repeats; STR: Short tandem repeat; BS: Bootstrap support; F: Forward; R: Reverse; C: Complement; p: Palindromic; PRICE: Paired-read iterative contig extension; SRA: Short read archive.}

\section{Supplementary Information}

The online version contains supplementary material available at https://doi. org/10.1186/s43008-021-00081-z.

Additional file 1. Figure S1: Similarity of the Ophiocordyceps gracilis and $O$. sinensis mitogenomes. The sequences are connected by differ ent colors according to similar regions and similar lengths, with scored colors show $\mathrm{n}$ in the histograms. The blank area between the connecting lines in the figure indicates that the two species do not have any similarity and are currently allowed only an $8.7 \%$ identity. Score coloring: blue $\leq 0.25$, green $\leq 0.50$, orange $\leq 0.75$, red $>0.75$. Data obtained from Kang et al. (2017).

Additional file 2. Table S1: Gene features and organization of the Ophiocordyceps gracilis and $O$. sinensis mitogenomes.

Additional file 3. Table S2: tRNA feature and organization in the Ophiocordyceps gracilis mitogenome.

Additional file 4. Table S3: Gene order of the Ophiocordycipitaceae mitogenomes.

Additional file 5. Table S4: Codon usage analysis of two different Ophiocordyceps species. 
Additional file 6. Table S5: Distribution of repeat loci in the mitogenome of Ophiocordyceps gracilis and $O$. sinensis as revealed by REPuter.

Additional file 7. Table S6: Tandem repeats detected in the mitogenome of Ophiocordyceps gracilis and 0 . sinensis.

Additional file 8. Table S7: Microsatellite DNA in Ophiocordyceps gracilis and $O$. sinensis mitogenome.

Additional file 9. Table S8: Introns in the Ophiocordyceps gracilis mitogenome.

Additional file 10. Table S9: Mitogenome information used for phylogenetic analysis.

\section{Acknowledgements}

We would like to thank Dr. GuiQi Bi (Agricultural Genomics Institute at Shenzhen, Chinese Academy of Agricultural Sciences, Shenzhen, China) for excellent technical guidance and precise comments in mitogenome assembly. We thank Dr. ChengJie Chen of South China Agricultural University for excellent technical assistance in bioinformatics and Tbtools usage. We also thank Falcon Scientific Editing (https://falconediting.com) for proofreading the English language in this paper.

\section{Authors' contributions}

FS and LH conceived and designed the experiments. AA, $H Z, R K$, and MD performed all experiments and data curation. AA and YW performed experimental data analysis and writing of the original manuscript. Modification and improvement of the manuscript were done by AA, YW, YJ, FS, and LH. All authors read and approved the final manuscript.

\section{Funding}

This work was supported by the Middle-aged and Young Teachers' Basic Ability Promotion Project of Guangxi (No. 2020KY01008), Graduate Research and Innovation Projects of Xinjiang Province(XJ2020G048), National Natural Science Foundation of China (32060007, 31860077), Natural Science Foundation of Xinjiang Province (2016D01C039), China Postdoctoral Science Foundation (2017M613017), and High-level introduction of talents research start-up fund of Guangxi University (A3130051004)

\section{Availability of data and materials}

All data generated or analyzed during this study are included in this published article. The authors promise the availability of supporting data.

\section{Declarations}

Ethics approval and consent to participate

Not applicable.

\section{Consent for publication}

Not applicable.

\section{Competing interests}

The authors declare no competing interests.

\section{Author details}

'College of Life Science and Technology, Xinjiang University, Urumchi 830046, China. ${ }^{2}$ CAS Key Laboratory for Plant Diversity and Biogeography of East Asia, Kunming Institute of Botany, Chinese Academy of Sciences, Kunming 650201, China. ${ }^{3}$ Food Crops Research Institute, Yunnan Academy of Agricultural Sciences, Kunming 650205, China. ${ }^{4}$ College of Life Science and Technology, Guangxi University, Nanning 530004, China. ${ }^{5}$ Guangxi Research Center for Microbial and Enzyme Engineering Technology, Guangxi University, Nanning 530004, China.

Received: 4 September 2020 Accepted: 10 October 2021 Published online: 20 October 2021

\section{References}

Bankevich A, Nurk S, Antipov D, Gurevich AA, Dvorkin M, Kulikov AS, Lesin VM, Nikolenko SI, Pham S, Prjibelski AD, Pyshkin AV, Sirotkin AV, Vyahhi N, Tesler G, Alekseyev MA, Pevzner PA (2012) SPAdes: a new genome assembly algorithm and its applications to single-cell sequencing. J Comput Biol 19(5):455-477

Beier S, Thiel T, Munch T, Scholz U, Mascher M (2017) MISA-web: a web server for microsatellite prediction. Bioinformatics 33(16):2583-2585

Borstler B, Raab PA, Thiery O, Morton JB, Redecker D (2008) Genetic diversity of the arbuscular mycorrhizal fungus Glomus intraradices as determined by mitochondrial large subunit rRNA gene sequences is considerably higher than previously expected. New Phytol 180(2):452-465

Bullerwell CE, Lang BF (2005) Fungal evolution: the case of the vanishing mitochondrion. Curr Opin Microbiol 8(4):362-369

Burger G, Gray MW, Lang BF (2003) Mitochondrial genomes: anything goes. Trends Genet 19(2):709-716

Chen CJ, Chen H, Zhang Y, Thomas HR, Frank MH, He YH, Xia R (2020) TBtools: an integrative toolkit developed for interactive analyses of big biological data. Mol Plant 13(8):1194-1202

Chen HX, Sun SC, Norenburg JL, Sundberg P (2014) Mutation and selection cause codon usage and bias in mitochondrial genomes of ribbon worms (Nemertea). PLoS ONE 9:e85631

Conant GC, Wolfe KH (2008) GenomeVx: simple web-based creation of editable circular chromosome maps. Bioinformatics 24(6):861-862

Dai LS, Kausar S, Abbas MN, Wang TT (2018) Complete sequence and characterization of the Ectropis oblique mitochondrial genome and its phylogenetic implications. Int J Biol Macromol 107:1142-1150

Darling ACE, Mau B, Blattner FR, Perna NT (2004) Mauve: multiple alignment of conserved genomic sequence with rearrangements. Genome Res 14(7):1394-1404

Darzentas N (2010) Circoletto: visualizing sequence similarity with Circos. Bioinformatics 26(20):2620-2621

Deng YJ, Zhang QH, Ming R, Lin LJ, Lin XZ, Lin YY, Li X, Xie BG, Wen ZQ (2016) Analysis of the mitochondrial genome in Hypomyces aurantius reveals a novel twintron complex in fungi. Int J Mol Sci 17(7):1049

Ferandon C, Xu J, Barroso G (2013) The 135 kbp mitochondrial genome of Agaricus bisporus is the largest known eukaryotic reservoir of group I introns and plasmid-related sequences. Fungal Genetice Biol 55:85-91

Garner TWJ (2002) Genome size and microsatellites: the effect of nuclear size on amplification potential. Genome 45(1):212-215

Himmelstrand K, Olson A, Brandstrom During M, Karlsson M, Stenlid J (2014) Intronic and plasmid-derived regions contribute to the large mitochondrial genome sizes of Agaricomycetes. Curr Genet 60(4):303-313

Huang F, Li Y, Chen X (2017) The complete mitochondrial genome of a medicinal fungus Tolypocladium ophioglossoides. Mitochondrial DNA Part B 2(1):95-96

Husami A, Slone J, Brown J, Bromwell M, Valencia CA, Huang T (2020) Clinical utility of whole genome sequencing for the detection of mitochondrial genome mutations. J Genet Genomics 47:167-169

Jalalzadeh B, Carras SI, Cyril F, Callac P, Farsi M, Savoie JM, Barroso G (2015) The intraspecific variability of mitochondrial genes of Agaricus bisporus reveals an extensive group I intron mobility combined with low nucleotide substitution rates. Curr Genet 61:87-102

Johnston IG, Williams BP (2016) Evolutionary inference across eukaryotes identifies specific pressures favoring mitochondrial gene retention. Cell Syst 2(2):101-111

Jung PP, Friedrich A, Reisser C, Hou J, Schacherer J (2012) Mitochondrial genome evolution in a single protoploid yeast species. G3 Genes Genomes Genetics 2(9):1103-1111

Kang XC, Hu LQ, Shen PY, Li R, Liu DB (2017) SMRT sequencing revealed mitogenome characteristic and mitogenome-wide DNA modification pattern in Ophiocordyceps sinensis. Front Microbiol 8:1422

Katoh K, Rozewicki J, Yamada KD (2017) MAFFT online service: multiple sequence alignment, interactive sequence choice and visualization. Briefings Bioinform 20(4):1160-1166

Kirchner S, Ignatova Z (2014) Emerging roles of tRNA in adaptive translation, signalling dynamics and disease. Nat Rev Genet 16(2):98-112

Kumar S, Stecher G, Tamura K (2016) MEGA7: molecular evolutionary genetics analysis version 7.0 for bigger datasets. Mol Biol Evol 33(7):1870-1874 
Kurtz S, Choudhuri JV, Ohlebusch E, Schleiermacher C, Stoye J, Giegerich R (2001) REPuter: the manifold applications of repeat analysis on a genomic scale. Nucleic Acids Res 29(22):4633-4642

Lagesen K, Hallin P, Rodland EA, Staerfeldt HH, Rognes T, Ussery DW (2007) RNAmmer: consistent and rapid annotation of ribosomal RNA genes. Nucleic Acids Res 35(9):3100-3108

Lang BF, Laforest MJ, Burger G (2007) Mitochondrial introns: a critical view. Trends in Genet 23(3):119-125

Liang S (2018) Missing pieces in the story of a caterpillar fungus Ophiocordyceps sinensis. IMA Fungus 9:75-77

Li DD, Zhang GD, Huang LD, Wang YB, Yu H (2019a) Complete mitochondrial genome of the important entomopathogenic fungus Cordyceps tenuipes (Hypocreales, Cordycipitaceae). Mitochondrial DNA Part B 4(1):1329-1331

Li JP, Zhang GD, Yu H, Huang LD, Zeng WB, Wang YB (2020a) Complete mitochondrial genome of the important bio-control fungus Purpureocillium lilacinum (Ophiocordycipitaceae, Hypocreales) and its phylogenetic analysis. Mitochondrial DNA Part B 5(1):240-242

Li Q, Wang Q, Chen C, Jin X, Chen Z, Xiong C, Li P, Zhao J, Huang W (2018) Characterization and comparative mitogenomic analysis of six newly sequenced mitochondrial genomes from ectomycorrhizal fungi (Russula) and phylogenetic analysis of the Agaricomycetes. Int J Biol Macromol 119:792-802

Li Q, Wang Q, Jin X, Chen Z, Xiong C, Li P, Zhao J, Huang W (2019b) Characterization and comparison of the mitochondrial genomes from two Lyophyllum fungal species and insights into phylogeny of Agaricomycetes. Int J Biol Macromol 121:364-372

Li Q, Ren YH, Xiang DB, Shi XD, Zhao JL, Peng LX, Zhao G (2020b) Comparative mitogenome analysis of two ectomycorrhizal fungi (Paxillus) reveals gene rearrangement, intron dynamics, and phylogeny of basidiomycetes. IMA Fungus 11:12

Li Y, Wang XL, Jiao L, Jiang Y, Li H, Jiang SP, Lhosumtseiring N, Fu SZ, Dong CH, Zhan Y, Yao YJ (2011) A survey of the geographic distribution of Ophiocordyceps sinensis. J Microbiol 49(6):913-919

Li Y, Hu XD, Yang RH, Hsiang T, Wang K, Liang DQ, Liang F, Cao DM, Zhou F, Wen G, Yao YJ (2015) Complete mitochondrial genome of the medicinal fungus Ophiocordyceps sinensis. Sci Rep 5:13892

Lin R, Liu C, Shen B, Bai M, Ling J, Chen G (2015) Analysis of the complete mitochondrial genome of Pochonia chlamydosporia suggests a close relationship to the invertebrate-pathogenic fungi in Hypocreales. BMC Microbiol 15:5

Lowe TM, Chan PP (2016) Trnascan-se on-line: integrating search and context for analysis of transfer rna genes. Nucleic Acids Res 44:W54-W57

Marioni JC, Mason CE, Mane SM, Stephens M, Gilad Y (2008) RNA-seq: an assessment of technical reproducibility and comparison with gene expression arrays. Genome Res 18(9):1509-1517

Nadimi M, Daubois L, Hijri M (2016) Mitochondrial comparative genomics and phylogenetic signal assessment of mtDNA among arbuscular mycorrhizal fungi. Mol Phylogenet Evol 98:74-83

Okonechnikov K, Golosova O, Fursov M (2012) Unipro UGENE: a unified bioinformatics toolkit. Bioinformatics 28(8):1166-1167

Paquin B, Laforest MJ, Forgetet L, Roewer I, Wang Z, Longcore J, Lang BF (1997) The fungal mitochondrial genome project: evolution of fungal mitochondrial genomes and their gene expression. Curr Genet 31(5):380-395

Qin Z, Yang YJ, Kang LL, Yan S, Cho K, Cai XY, Lu Y, Zheng HX, Zhu DC, Fei DM, Li SL, Jin L, Li H, Genographic consortium (2010) A mitochondrial revelation of early human migrations to the Tibetan Plateau before and after the last glacial maximum. Am J Phys Anthropol 143(4):555-569

Quandt CA, Kepler RM, Gams W, Araújo JPM, Ban S, Evans HC, Hughes D, Humber R, Hywel-Jones N, Li Z, Luangsa-Ard JJ, Rehner SA, Sanjuan T, Sato H, Shrestha B, Sung GH, Yao YJ, Zare R, Spatafora JW (2014) Phylogeneticbased nomenclatural proposals for Ophiocordycipitaceae (Hypocreales) with new combination in Tolypocladium. IMA Fungus 5:121-134

Ronquist F, Teslenko M, Mark PVD, Ayres DL, Darling A, Hohna S, Larget B, Liu L, Suchard MA, Huelsenbeck JP (2012) MrBayes 32: efficient Bayesian phylogenetic inference and model choice across a large model space. Syst Biol 61(3):539-542

Ruby JG, Bellare P, DeRisi JL (2013) PRICE: software for the targeted assembly of components of (Meta) genomic sequence data. G3 Genes Genomes Genetics 3(5):865-880
Saldanha R, Mohr G, Belfort M, Lambowitz AM (1993) Group I and group II introns. FASEB J 7(1):15-24

Sandor S, Zhang Y, Xu J (2018) Fungal mitochondrial genomes and genetic polymorphisms. Appl Microbiol Biotechnol 102:9433-9448

Serrão VHB, Silva IR, da Silva MTA, Scortecci JF, de Freitas FA, Thiemann OH (2018) The unique tRNA ${ }^{\mathrm{Sec}}$ and its role in selenocysteine biosynthesis. Amino Acids 50(9):1145-1167

Sosa-Gómez DR, Humber RA, Hodge KT, Binneck E, Silva-Brandao KLD (2009) Variability of the mitochondrial SSU rDNA of Nomuraea species and other entomopathogenic fungi from Hypocreales. Mycopathologia 167:145-154

Stamatakis A (2014) RAxML version 8: a tool for phylogenetic analysis and post-analysis of large phylogenies. Bioinformatics 30(9):1312-1313

Sung GH, Hywel-Jones NL, Sung JM, Luangsa-ard JJ, Shrestha B, Spatafora JW (2007) Phylogenetic classification of Cordyceps and the clavicipitaceous fungi. Stud Mycol 57:5-59

Torriani SFF, Penselin D, Knogge W, Felder M, Taudien S, Platzer M, Mcdonald BA, Brunner PC (2014) Comparative analysis of mitochondrial genomes from closely related Rhynchosporium species reveals extensive intron invasion. Fungal Genet Biol 62(1):34-42

Valach M, Burger G, Gray MW, Lang BF (2014) Widespread occurrence of organelle genome-encoded $5 \mathrm{~S}$ rRNAs including permuted molecules. Nucleic Acids Res 42(22):13764-13777

Vargas-Rodriguez O, Englert M, Merkuryev A, Mukai T, Soll D (2018) Recoding of the selenocysteine UGA codon by cysteine in the presence of a noncanonical tRNA ${ }^{\text {Cys }}$ and elongation factor SelB. RNA Biol 15(4-5):471-479

Wang L, Zhang S, Li JH, Zhang YJ (2018) Mitochondrial genome, comparative analysis and evolutionary insights into the entomopathogenic fungus Hirsutella thompsonii. Environ Microbiol 20(9):3393-3405

Wang N, Zhang Y, Hussain M, Li K, Xiang M, Liu X (2016) The mitochondrial genome of the nematode endoparasitic fungus Hirsutella rhossiliensis. Mitochondrial DNA Part B 1(1):114-115

Wang X, Wang Y, Yao W, Shen J, Chen M, Gao M, Ren J, Li Q, Liu N (2020a) The $256 \mathrm{~kb}$ mitochondrial genome of Clavaria fumosa is the largest among phylum Basidiomycota and is rich in introns and intronic ORFs. IMA Fungus 11:26

Wang YB, Wang Y, Fan Q, Duan DE, Zhang GD, Dai RQ, Dai YD, Zeng WB, Chen ZH, Li DD, Tang DX, Xu ZH, Sun T, Nguyen TT, Tran NL, Dao VM, Zhang CM, Huang LD, Liu YJ, Zhang XM, Yang DR, Sanjuan T, Liu XZ, Yang ZL, Yu H (2020b) Multigene phylogeny of the family Cordycipitaceae (Hypocreales): new taxa and the new systematic position of the Chinese cordycipitoid fungus Paecilomyces hepiali. Fungal Divers 103:1-46

Xu J, Yan Z, Guo H (2009) Divergence, hybridization, and recombination in the mitochondrial genome of the human pathogenic yeast Cryptococcus gattii. Mol Ecol 18(12):2628-2642

Zhang S, Zhang YJ (2019) Proposal of a new nomenclature for introns in protein-coding genes in fungal mitogenomes. IMA Fungus 10:15

Zhang S, Zhang YJ (2020) Complete mitogenome of the entomopathogenic fungus Tolypocladium cylindrosporum. Mitochondrial DNA Part B 5(1):680-682

Zhang Y, Zhang S, Zhang G, Liu X, Wang C, Xu J (2015a) Comparison of mitochondrial genomes provides insights into intron dynamics and evolution in the caterpillar fungus Cordyceps militaris. Fungal Genet Biol 77:95-107

Zhang YJ, Zhang S, Liu XZ (2015b) The complete mitochondrial genome of the nematode endoparasitic fungus Hirsutella minnesotensis. Mitochondrial DNA Part A 27(4):2693-2694

Zhang YJ, Zhang HY, Liu XZ, Zhang S (2017a) Mitochondrial genome of the nematode endoparasitic fungus Hirsutella vermicola reveals a high level of synteny in the family Ophiocordycipitaceae. Appl Microbiol Biotechnol 101:3295-3304

Zhang YJ, Yang XQ, Zhang S, Humber RA, Xu J (2017b) Genomic analyses reveal low mitochondrial and high nuclear diversity in the cyclosporinproducing fungus Tolypocladium inflatum. Appl Microbiol Biotechnol 101(23-24):8517-8531

\section{Publisher's Note}

Springer Nature remains neutral with regard to jurisdictional claims in published maps and institutional affiliations. 